Elect. Comm. in Probab. 13 (2008), 325-336

\title{
ON THE RELATIONSHIP BETWEEN SUBORDINATE KILLED AND KILLED SUBORDINATE PROCESSES
}

\author{
RENMING SONG \\ Department of Mathematics, University of Illinois, Urbana, IL 61801 \\ email: rsong@math.uiuc.edu \\ ZORAN VONDRAČEK ${ }^{1}$ \\ Department of Mathematics, University of Zagreb, Bijenička 30, 10000 Zagreb, Croatia \\ email: vondra@math.hr
}

Submitted 25 January 2008, accepted in final form 16 June 2008

AMS 2000 Subject classification: Primary 60J25; secondary 60J45, 60J75

Keywords: Markov process, subordination, killing, resurrection

\section{Abstract}

We study the precise relationship between the subordinate killed and killed subordinate processes in the case of an underlying Hunt process, and show that, under minimal conditions, the former is a subprocess of the latter obtained by killing at a terminal time. Moreover, we also show that the killed subordinate process can be obtained by resurrecting the subordinate killed one at most countably many times.

\section{Introduction}

Let $X$ be a strong Markov process on a state space $E$. In this paper we will be interested in two types of probabilistic transformations of $X$. The first one is subordination of $X$ via an independent subordinator $T$ giving a Markov process $Y=\left(Y_{t}: t \geq 0\right)$ on $E$ defined by $Y_{t}=X\left(T_{t}\right)$. The other transformation is killing $X$ upon exiting an open subset $D$ of $E$. The resulting process $X^{D}$ is defined by $X_{t}^{D}=X_{t}$ for $t<\tau_{D}=\inf \left\{t>0: X_{t} \notin D\right\}$, and $X_{t}^{D}=\partial$ (the cemetery) otherwise. Now one can kill $Y$ upon exiting $D$ giving the process $Y^{D}$, and also subordinate $X^{D}$ by the same subordinator $T$ giving the process that we will denote by $Z^{D}$. Both processes are Markov with the same state space $D$. The process $Y^{D}$ is called the killed subordinate process (first subordination, then killing), while $Z^{D}$ is called the subordinate killed process (first killing, then subordination). It is an interesting problem to investigate the precise relationship between these two processes. This question can be traced back to [4] in the case when $X$ is a Brownian motion and $T$ a stable subordinator. In this context it was addressed in [10] where by use of pathwise approach it was shown that the semigroup of $Z^{D}$ (subordinate killed) is subordinate to the semigroup of $Y^{D}$ (killed subordinate). Recently, by

\footnotetext{
${ }^{1}$ THE RESEARCH OF THIS AUTHOR IS SUPPORTED IN PART BY THE MZOS GRANT 037-03727902801 OF THE REPUBLIC OF CROATIA.
} 
use of Dirichlet form techniques, He and Ying gave in [5] an answer in a general setting of symmetric Borel right processes on a Lusin space $E$. Again, the semigroup of $Z^{D}$ is subordinate to the semigroup of $Y^{D}$. The general theory then implies that $Z^{D}$ can be obtained by killing $Y^{D}$ via a multiplicative functional.

The goal of this paper is to give (in our opinion) the complete description of the relationship between $Z^{D}$ and $Y^{D}$ in the context of a Hunt process $X$ (not necessarily symmetric) on a locally compact second countable Hausdorff space $E$. By defining $X$ and the subordinator $T$ on appropriate path spaces, and considering all relevant processes on the product of these path spaces, we show that $Z^{D}$ is obtained by killing $Y^{D}$ at an identifiable terminal time with respect to a filtration making $Y$ a strong Markov process. Note that killing at a terminal time is a special case of killing by a multiplicative functional, but clearly more transparent. Moreover, we go a step further and show that the process $Y^{D}$ can be recovered from $Z^{D}$ by resurrecting the latter at most countably many times. This easily follows from our setting in which both $Z^{D}$ and $Y^{D}$ are described explicitly in terms of the underlying Hunt process $X$ and the subordinator $T$. We also compute the resurrection kernel (given, implicitly, in [5]). Having the resurrection kernel, one can now start from any process with the same distribution as $Z^{D}$ and use Meyer's resurrection procedure described in [7] to construct a process with the distribution of $Y^{D}$.

The paper is organized as follows: In the next section we precisely describe our setting. In Section 3 we give a description of the relationship between subordinate killed and killed subordinate processes. In Section 4 the resurrection kernel is computed. In the last section, as an application, we give sufficient conditions for $Y$ to be not on the boundary $\partial D$ at the exit time from $D$.

\section{Setting and notation}

Let $E$ be a locally compact second countable Hausdorff space and let $\mathcal{E}$ be the corresponding Borel $\sigma$-algebra. Further, let $\Omega_{1}$ be the set of all functions $\omega_{1}:[0, \infty) \rightarrow E$ which are right continuous and have left limits. For each $t \geq 0$, let $X_{t}: \Omega_{1} \rightarrow E$ be defined by $X_{t}\left(\omega_{1}\right)=\omega_{1}(t)$. The shift operator $\vartheta_{t}^{1}: \Omega_{1} \rightarrow \Omega_{1}$ is defined by $\vartheta_{t}^{1} \omega_{1}(\cdot)=\omega_{1}(t+\cdot)$. Let $\mathbb{F}^{0}=\left(\mathcal{F}_{t}^{0}: t \geq 0\right)$, $\mathcal{F}_{t}^{0}=\sigma\left(X_{s}: 0 \leq s \leq t\right)$, be the natural filtration generated by the process $X=\left(X_{t}: t \geq 0\right)$, and let $\mathcal{F}_{t+}^{0}=\cap_{s>t} \mathcal{F}_{s}^{0}$. Also, let $\mathcal{F}=\sigma\left(X_{t}: t \geq 0\right)$. We assume that $\left(\mathbb{P}_{1}^{x}: x \in E\right)$ is a family of probability measures on $\left(\Omega_{1}, \mathcal{F}\right)$ such that $\left(X_{t}, \mathbb{P}_{1}^{x}\right)$ is a strong Markov process. Let $\mathbb{F}=\left(\mathcal{F}_{t}: t \geq 0\right)$ be the usual augmentation of the natural filtration $\mathbb{F}^{0}$. From now on we assume that $X=\left(\Omega_{1}, \mathcal{F}, \mathcal{F}_{t}, X_{t}, \vartheta_{t}^{1}, \mathbb{P}_{1}^{x}\right)$ is a Hunt process with the state space $(E, \mathcal{E})$.

Let $\Omega_{2}$ be the set of all functions $\omega_{2}:[0, \infty) \rightarrow[0, \infty)$ which are right continuous and have left limits. For each $t \geq 0$, let $T_{t}: \Omega_{2} \rightarrow[0, \infty)$ be defined by $T_{t}\left(\omega_{2}\right)=\omega_{2}(t)$. The shift operator $\vartheta_{t}^{2}: \Omega_{2} \rightarrow \Omega_{2}$ is defined by $\vartheta_{t}^{2} \omega_{2}(\cdot)=\omega_{2}(t+\cdot)$. Let $\mathbb{G}^{0}=\left(\mathcal{G}_{t}^{0}: t \geq 0\right), \mathcal{G}_{t}^{0}=\sigma\left(T_{s}: 0 \leq s \leq t\right)$, be the natural filtration generated by the process $T=\left(T_{t}: t \geq 0\right)$, and let $\mathcal{G}_{t+}^{0}=\cap_{s}>{ }_{t} \mathcal{G}_{s}^{0}$. Also, let $\mathcal{G}=\sigma\left(T_{t}: t \geq 0\right)$. We assume that $\left(\mathbb{P}_{2}^{y}: y \in[0, \infty)\right)$ is a family of probability measures on $\left(\Omega_{2}, \mathcal{G}\right)$ such that $\left(T_{t}, \mathbb{P}_{2}^{y}\right)$ is an increasing Lévy process. In particular, we assume that under $\mathbb{P}_{2}:=\mathbb{P}_{2}^{0}$, the law of $T_{t}$ is given by

$$
\mathbb{E}_{2}\left(\exp -\lambda T_{t}\right)=\exp (-t \phi(\lambda))
$$

where

$$
\phi(\lambda)=b \lambda+\int_{(0, \infty)}\left(1-e^{-\lambda x}\right) \Pi(d x)
$$


Here $b \geq 0$ is the drift, and $\Pi$ the Lévy measure of the subordinator $T$. Further, let $U(d y)$ denote the potential measure of $T$ under $\mathbb{P}_{2}$ :

$$
U(d y)=\mathbb{E}_{2} \int_{0}^{\infty} 1_{\left(T_{t} \in d y\right)} d t .
$$

For $y>0$, let $\sigma_{y}=\inf \left\{t>0: T_{t}>y\right\}$ be the first passage time of $T$ across the level $y$. Then $\sigma_{y}$ is a $\left(\mathcal{G}_{t+}^{0}\right)$-stopping time and the following identity holds true for all $t>0$ and $y>0$ :

$$
\left\{T_{t}<y\right\}=\left\{\sigma_{y}>t\right\} .
$$

Let $\Omega=\Omega_{1} \times \Omega_{2}$, and, for any $x \in E$ and $y \in[0, \infty)$, let $\mathbb{P}^{x, y}=\mathbb{P}_{1}^{x} \times \mathbb{P}_{2}^{y}$ be the product probability measure on $\mathcal{H}=\mathcal{F} \times \mathcal{G}$. The probability $\mathbb{P}^{x, 0}$ will be denoted as $\mathbb{P}^{x}$. The elements of $\Omega$ are denoted by $\omega=\left(\omega_{1}, \omega_{2}\right)$. For each $t \geq 0$ we define the shift operator $\theta_{t}: \Omega \rightarrow \Omega$ by

$$
\theta_{t}(\omega)(\cdot)=\theta_{t}\left(\omega_{1}, \omega_{2}\right)(\cdot):=\left(\omega_{1}\left(\omega_{2}(t)+\cdot\right), \omega_{2}(t+\cdot)-\omega_{2}(t)\right) .
$$

We will occasionally write $\theta_{t}(\omega)=\left(\theta_{t}^{1}(\omega), \theta_{t}^{2}(\omega)\right)$. Note that for $s, t \geq 0$ we have

$$
\begin{aligned}
\theta_{s}\left(\theta_{t} \omega\right) & =\theta_{s}\left(\omega_{1}\left(\omega_{2}(t)+\cdot\right), \omega_{2}(t+\cdot)-\omega_{2}(t)\right) \\
& =\left(\omega_{1}\left(\omega_{2}(t)+\left(\omega_{2}(t+s)-\omega_{2}(t)\right)+\cdot\right),\left(\omega_{2}(t+s+\cdot)-\omega_{2}(t)\right)-\left(\omega_{2}(t+s)-\omega_{2}(t)\right)\right) \\
& =\left(\omega_{1}\left(\omega_{2}(t+s)+\cdot\right), \omega_{2}(t+s+\cdot)-\omega_{2}(t+s)\right) \\
& =\theta_{t+s}(\omega) .
\end{aligned}
$$

The subordinate process $Y=\left(Y_{t}: t \geq 0\right)$ with the state space $(E, \mathcal{E})$ is defined on $\Omega$ by $Y_{t}(\omega):=X_{T_{t}\left(\omega_{2}\right)}\left(\omega_{1}\right)=\omega_{1}\left(\omega_{2}(t)\right)$. Note that

$$
\begin{aligned}
Y_{s}\left(\theta_{t} \omega\right) & =\omega_{1}\left(\omega_{2}(t)+\left(\omega_{2}(t+s)-\omega_{2}(t)\right)\right) \\
& =\omega_{1}\left(\omega_{2}(t+s)\right)=Y_{s+t}(\omega) .
\end{aligned}
$$

Following [3] we introduce the following filtration: For $t \geq 0$ let

$$
\mathcal{S}_{t}=\left\{A^{1} \times\left(A^{2} \cap\left\{T_{t} \geq u\right\}\right): A^{1} \in \mathcal{F}_{u}^{0}, A^{2} \in \mathcal{G}_{t}^{0}, u \geq 0\right\},
$$

and let $\mathcal{H}_{t}=\sigma\left(\mathcal{S}_{t}\right)$. Then $\mathbb{H}=\left(\mathcal{H}_{t}: t \geq 0\right)$ is a filtration on $\Omega$ such that for all $t \geq 0$, $\sigma\left(Y_{s}: 0 \leq s \leq t\right) \subset \mathcal{H}_{t}$.

Remark 2.1. Suppose that $S$ is a function defined on, say, $\Omega_{1}$. By abusing notation we will regard $S$ as being defined on $\Omega$ by $S\left(\omega_{1}, \omega_{2}\right)=S\left(\omega_{1}\right)$. We use the same convention if $S$ is a function defined on $\Omega_{2}$.

The following results are proved in [3]:

Proposition 2.2. (i) ([3], p.65) If $S$ is an $\left(\mathcal{F}_{t+}^{0}\right)$-stopping time, then $\left\{S \leq T_{t}\right\} \in \mathcal{H}_{t+}$.

(ii) ([3], p.66) If $S$ is an $\left(\mathcal{H}_{t+}\right)$-stopping time, then

(a) for each $\omega_{1}, S\left(\omega_{1}, \cdot\right)$ is an $\left(\mathcal{G}_{t+}^{0}\right)$-stopping time;

(b) for each $\omega_{2}, T_{S\left(\cdot, \omega_{2}\right)}\left(\omega_{2}\right)$ is an $\left(\mathcal{F}_{t+}^{0}\right)$-stopping time.

(iii) ([3], p. 67) The subordinate process $Y=\left(\Omega, \mathcal{H}, \mathcal{H}_{t+}, Y_{t}, \mathbb{P}^{x, y}\right)$ is a strong Markov process. 
In the next result we prove that the subordinate process $Y$ is quasi-left-continuous.

Proposition 2.3. Let $\left(S_{n}: n \geq 1\right)$ be an increasing sequence of $\left(\mathcal{H}_{t+}\right)$-stopping times, and let $S=\lim _{n \rightarrow \infty} S_{n}$. Then $\lim _{n \rightarrow \infty} Y_{S_{n}}=Y_{S}, \mathbb{P}^{x}$-a.s. on $\{S<\infty\}$ for every $x \in E$.

Proof. Without loss of generality we assume that $S<\infty, \mathbb{P}^{x}$-a.s., for every $x \in E$. Let $A=\left\{\omega=\left(\omega_{1}, \omega_{2}\right): \lim _{n} T_{S_{n}\left(\omega_{1}, \omega_{2}\right)}\left(\omega_{2}\right)=T_{S\left(\omega_{1}, \omega_{2}\right)}\left(\omega_{2}\right)\right\}$, and let $A_{\omega_{1}}$ be the $\omega_{1}$-section of $A$. For each fixed $\omega_{1}$, it follows from Proposition $2.2\left(\right.$ ii) (a) that $S_{n}\left(\omega_{1}, \cdot\right)$ is a $\left(\mathcal{G}_{t+}^{0}\right)$-stopping time, hence by quasi-left-continuity of the subordinator $T$, we have that $\mathbb{P}_{2}\left(A_{\omega_{1}}\right)=1$. Thus by Fubini's theorem we have that $\mathbb{P}^{x}(A)=1$, for every $x \in E$.

Let $\widetilde{T}\left(\omega_{1}, \omega_{2}\right)=\lim _{n \rightarrow \infty} T_{S_{n}\left(\omega_{1}, \omega_{2}\right)}\left(\omega_{2}\right)$. Since for each fixed $\omega_{2}, T_{S_{n}\left(\cdot, \omega_{2}\right)}\left(\omega_{2}\right)$ is an $\left(\mathcal{F}_{t+}^{0}\right)$ stopping time by Proposition $2.2\left(\right.$ ii) (b), it follows that $\widetilde{T}\left(\cdot, \omega_{2}\right)$ is also an $\left(\mathcal{F}_{t+}^{0}\right)$-stopping time. The assumptions $S<\infty \mathbb{P}^{x}$-a.s. implies that $\widetilde{T}\left(\cdot, \omega^{2}\right)<\infty, \mathbb{P}_{1}^{x}$-a.s., for every $x \in E$. By the quasi-left-continuity of the process $X$ we obtain that

$$
\lim _{n \rightarrow \infty} X_{T_{S_{n}\left(\omega_{1}, \omega_{2}\right)}}\left(\omega_{1}\right)=X_{\widetilde{T}\left(\omega_{1}, \omega_{2}\right)}\left(\omega_{1}\right) \quad \mathbb{P}_{1}^{x} \text {-a.e. } \omega_{1} .
$$

Let $B=\left\{\omega=\left(\omega_{1}, \omega_{2}\right): \lim _{n \rightarrow \infty} X_{T_{S_{n}}}(\omega)=X_{\widetilde{T}}(\omega)\right\}$. Again by using Fubini's theorem, it follows that $\mathbb{P}^{x}(B)=1$ for every $x \in E$. Therefore, $\mathbb{P}^{x}(A \cap B)=1$, and for $\omega \in A \cap B$ it holds that $\lim _{n \rightarrow \infty} Y_{S_{n}}(\omega)=\lim _{n \rightarrow \infty} X_{T_{S_{n}}}(\omega)=X_{\widetilde{T}}(\omega)=X_{T_{S}}(\omega)=Y_{S}(\omega)$.

Lemma 2.4. Let $S$ be an $\left(\mathcal{F}_{t+}^{0}\right)$-stopping time. Then $\sigma_{S}=\inf \left\{t>0: T_{t}>S\right\}$ is an $\left(\mathcal{H}_{t+}\right)$-stopping time.

Proof. By (2.1) we have that $\left\{\sigma_{S} \leq t\right\}=\left\{T_{t} \geq S\right\}$. The claim now follows from Proposition $2.2(\mathrm{i})$.

Let $D$ be an open subset of $E$, and let $\tau_{D}=\inf \left\{t>0: X_{t} \notin D\right\}$ be the first exit time of $X$ from $D$. We assume for simplicity that $\mathbb{P}_{1}^{x}\left(\tau_{D}<\infty\right)=1$ for all $x \in E$. By the previous lemma it follows that $\sigma_{\tau_{D}}$ is an $\left(\mathcal{H}_{t+}\right)$-stopping time. In the next lemma we prove that it is also a terminal time with respect to $\left(\mathcal{H}_{t+}\right)$.

Lemma 2.5. For every $t \geq 0, \sigma_{\tau_{D}}=t+\sigma_{\tau_{D}} \circ \theta_{t}$ on $\left\{t<\sigma_{\tau_{D}}\right\}$.

Proof. First note that $\sigma_{\tau_{D}}(\omega)=\sigma_{\tau_{D}}\left(\omega_{1}, \omega_{2}\right)=\inf \left\{t>0: \omega_{2}(t)>\tau_{D}\left(\omega_{1}\right)\right\}$. Further, by (2.1), $\left\{t<\sigma_{\tau_{D}}\right\}=\left\{T_{t}<\tau_{D}\right\}=\left\{\left(\omega_{1}, \omega_{2}\right): \omega_{2}(t)<\inf \left\{u>0: \omega_{1}(u) \notin D\right\}\right\}$. Therefore, on $\left\{t<\sigma_{\tau_{D}}\right\}$,

$$
\begin{aligned}
& \sigma_{\tau_{D}}\left(\theta_{t} \omega\right)=\inf \left\{s>0: \theta_{t}^{2} \omega_{2}(s)>\tau_{D}\left(\theta_{t} \omega\right)\right\} \\
& \quad=\inf \left\{s>0: \omega_{2}(t+s)-\omega_{2}(t)>\inf \left\{u>0:\left(\theta_{t}^{1} \omega\right)(u) \notin D\right\}\right\} \\
& \quad=\inf \left\{s>0: \omega_{2}(t+s)>\omega_{2}(t)+\inf \left\{u>0: \omega_{1}\left(\omega_{2}(t)+u\right) \notin D\right\}\right\} \\
& \quad=\inf \left\{s>0: \omega_{2}(t+s)>\inf \left\{u>0: \omega_{1}(u) \notin D\right\}\right\},
\end{aligned}
$$

where the last line follows from $\omega_{2}(t)<\inf \left\{u>0: \omega_{1}(u) \notin D\right\}$. Hence,

$$
\begin{aligned}
t+\left(\sigma_{\tau_{D}} \circ \theta_{t}\right)(\omega) & =t+\inf \left\{s>0: \omega_{2}(t+s)>\tau_{D}(\omega)\right\} \\
& =\inf \left\{s>0: \omega_{2}(s)>\tau_{D}(\omega)\right\}=\sigma_{\tau_{D}}(\omega) .
\end{aligned}
$$

We also record the fact that for each fixed $\omega_{2}, T_{\sigma_{\tau_{D}}\left(\cdot, \omega_{2}\right)}\left(\omega_{2}\right)$ is an $\left(\mathcal{F}_{t+}^{0}\right)$-stopping time. This follows from Proposition 2.2(ii)(b). Further, for each fixed $\omega_{2}, T_{\sigma_{\tau_{D}}\left(\cdot, \omega_{2}\right)}\left(\omega_{2}\right)$ is a function of $\tau_{D}$, and hence $\mathcal{F}_{\tau_{D}}^{0}$-measurable. 


\section{Subordinate killed and killed subordinate processes}

Let $\tau_{D}^{Y}=\inf \left\{t>0: Y_{t} \notin D\right\}$ be the first exit time of the subordinate process $Y$ from $D$. Then $\tau_{D}^{Y}$ is an $\left(\mathcal{H}_{t+}\right)$-stopping time. We note that even when $\mathbb{P}_{1}^{x}\left(\tau_{D}<\infty\right)=1$ for all $x \in D$, it may happen that $\tau_{D}^{Y}=\infty, \mathbb{P}^{x}$-a.s. for every $x \in D$. Indeed, let $X$ be a one-dimensional Brownian motion, $D=(-\infty, 0) \cup(0, \infty)$, and let $T$ be an $\alpha / 2$-stable subordinator with $0<\alpha<1$. The subordinate process $Y$ is an $\alpha$-stable process in $\mathbb{R}$. Since $0<\alpha<1$, points are polar for $Y$, and in particular, the hitting time to zero, which is precisely equal to $\tau_{D}^{Y}$, is infinite. Clearly, $\mathbb{P}_{1}^{x}\left(\tau_{D}<\infty\right)=1$.

The process $Y$ killed upon exiting $D$ is defined by

$$
Y_{t}^{D}:=\left\{\begin{array}{ll}
Y_{t}, & t<\tau_{D}^{Y} \\
\partial, & t \geq \tau_{D}^{Y}
\end{array}= \begin{cases}X_{T_{t}}, & t<\tau_{D}^{Y} \\
\partial, & t \geq \tau_{D}^{Y}\end{cases}\right.
$$

where $\partial$ is a cemetery point. We call $Y^{D}$ the killed subordinate process. Note that $Y^{D}$ is a strong Markov process with respect to the filtration $\left(\mathcal{H}_{t+}\right)$.

The other process that we are going to consider is obtained by killing $Y$ at the terminal time $\sigma_{\tau_{D}}$. Define

$$
Z_{t}^{D}:=\left\{\begin{array}{ll}
Y_{t}, & t<\sigma_{\tau_{D}} \\
\partial, & t \geq \sigma_{\tau_{D}}
\end{array}= \begin{cases}X_{T_{t}}, & T_{t}<\tau_{D} \\
\partial, & T_{t} \geq \tau_{D}\end{cases}\right.
$$

where the equality is a consequence of (2.1). Since $\sigma_{\tau_{D}}$ is a terminal time, it follows (similarly as in the proof of Theorem 12.23(i) of [9], p. 71) that $Z^{D}$ is also a strong Markov process with respect to the filtration $\left(\mathcal{H}_{t+}\right)$.

We can also introduce the process $X^{D}$ as the process $X$ killed upon exiting $D$. Clearly, if $T_{t}<\tau_{D}$, then $X_{T_{t}}^{D}=X_{T_{t}}$. This shows that $Z^{D}$ is in fact obtained by first killing $X$ as it exits $D$, and then by subordinating the killed process with the subordinator $T$. Therefore we call $Z^{D}$ the subordinate killed process.

Note that if $t<\sigma_{\tau_{D}}$, then $T_{t}<\tau_{D}$, and therefore $Y_{t}=X_{T_{t}} \in D$. This shows that $\sigma_{\tau_{D}} \leq \tau_{D}^{Y}$. As a consequence, we see that $Z^{D}$ can be obtained by killing $Y^{D}$ at the terminal time $\sigma_{\tau_{D}}$ :

$$
Z_{t}^{D}:= \begin{cases}Y_{t}^{D}, & t<\sigma_{\tau_{D}} \\ \partial, & t \geq \sigma_{\tau_{D}}\end{cases}
$$

For any nonnegative Borel function $f$ on $D$ let $Q_{t}^{D} f(x):=\mathbb{E}^{x}\left[f\left(Y_{t}^{D}\right)\right]$ be the semigroup of $Y^{D}$, and $R_{t}^{D} f(x):=\mathbb{E}^{x}\left[f\left(Z_{t}^{D}\right)\right]=\mathbb{E}^{x}\left[f\left(Y_{t}^{D}\right), t<\sigma_{\tau_{D}}\right]$ be the semigroup of $Z^{D}$. The following result is now obvious.

Proposition 3.1. The semigroup $\left(R_{t}^{D}: t \geq 0\right)$ is subordinate to the semigroup $\left(Q_{t}^{D}: t \geq 0\right)$ in the sense that for every nonnegative Borel function $f$ on $D$ it holds that $R_{t}^{D} f \leq Q_{t}^{D} f$.

Let $S_{1}=\sigma_{\tau_{D}}$ and define inductively $S_{n+1}=S_{n}+S_{1} \circ \theta_{S_{n}}$ with the convention that if $S_{n}=\tau_{D}^{Y}$, then for all $k>n, S_{k}=\tau_{D}^{Y}$. Then $\left(S_{n}: n \geq 1\right)$ is an increasing sequence of $\left(\mathcal{H}_{t+}\right)$-stopping times. The limit $S=\lim _{n \rightarrow \infty} S_{n}$ is an $\left(\mathcal{H}_{t+}\right)$-stopping time. Clearly, $S \leq \tau_{D}^{Y}$. The next proposition shows that these stopping times are in fact equal $\mathbb{P}^{x}$-a.s. for every $x \in D$.

Proposition 3.2. It holds that $\tau_{D}^{Y}=S, \mathbb{P}^{x}$-a.s. for every $x \in D$.

Proof. Let $A=\left\{\omega=\left(\omega_{1}, \omega_{2}\right): \lim _{n} T_{S_{n}\left(\omega_{1}, \omega_{2}\right)}\left(\omega_{2}\right)=T_{S\left(\omega_{1}, \omega_{2}\right)}\left(\omega_{2}\right)\right\}$. It was shown in Proposition 2.3 that $\mathbb{P}^{x}(A)=1$ for all $x \in E$. Therefore, there exists $\widetilde{\Omega}_{2} \subset \Omega_{2}$ with $\mathbb{P}_{2}\left(\widetilde{\Omega}_{2}\right)=1$ and such that $\mathbb{P}_{1}^{x}\left(A_{\omega_{2}}\right)=1$ for every $\omega_{2} \subset \widetilde{\Omega}_{2}$. Let us fix $\omega_{2} \in \widetilde{\Omega}_{2}$. If for some $n \geq 1$ it holds 
that $S_{n}\left(\cdot, \omega_{2}\right)=\tau_{D}^{Y}\left(\cdot, \omega_{2}\right)$ there is nothing to prove. Therefore we assume that $S_{1}\left(\cdot, \omega_{2}\right)<$ $S_{2}\left(\cdot, \omega_{2}\right)<\cdots<S\left(\cdot, \omega_{2}\right)$. By Proposition $\left.2.2, T_{S\left(\cdot, \omega_{2}\right.}\right)\left(\omega_{2}\right)$ and $\left.T_{S_{n}\left(\cdot, \omega_{2}\right.}\right)\left(\omega_{2}\right), n \in \mathbb{N}$, are $\left(\mathcal{F}_{t+}^{0}\right)$-stopping times. For $n \geq 1$ define

$$
\left.\tau_{n+1}=\inf \left\{t>T_{S_{n}\left(\cdot, \omega_{2}\right.}\right)\left(\omega_{2}\right): X_{t} \notin D\right\}
$$

Then $\tau_{n+1}$ is an $\left(\mathcal{F}_{t+}^{0}\right)$-stopping time. Moreover, we have that

$$
\left.\left.\left.T_{S_{1}\left(\cdot, \omega_{2}\right.}\right)\left(\omega_{2}\right) \leq \tau_{2} \leq T_{S_{s}\left(\cdot, \omega_{2}\right.}\right)\left(\omega_{2}\right) \leq \tau_{3} \leq \cdots \leq T_{S\left(\cdot, \omega_{2}\right.}\right)\left(\omega_{2}\right)
$$

Since $\mathbb{P}_{1}^{x}\left(A_{\omega_{2}}\right)=1$, we have that $\left.T_{S\left(\cdot, \omega_{2}\right.}\right)\left(\omega_{2}\right)=\uparrow \lim _{n \rightarrow \infty} \tau_{n}, \mathbb{P}_{1}^{x}$-a.s., and by the quasi-leftcontinuity of $X$, we conclude that $X_{\left.T_{S\left(\cdot, \omega_{2}\right.}\right)\left(\omega_{2}\right)}=\lim _{n \rightarrow \infty} X_{\tau_{n}}, \mathbb{P}_{1}^{x}$-a.s. on $\left\{T_{S\left(\cdot, \omega_{2}\right.}\right)\left(\omega_{2}\right)<$ $\infty\}=\left\{S\left(\cdot, \omega_{2}\right)<\infty\right\}$ for every $x \in D$. Since $X_{\tau_{n}} \notin D$, we conclude that $\mathbb{P}_{1}^{x}\left(X_{\left.T_{S\left(\cdot, \omega_{2}\right.}\right)\left(\omega_{2}\right)} \notin\right.$ $\left.\left.D, S\left(\cdot, \omega_{2}\right)<\infty\right)\right)=\mathbb{P}_{1}^{x}\left(S\left(\cdot, \omega_{2}\right)<\infty\right)$ for every $x \in D$. Since $\omega_{2} \in \widetilde{\Omega}_{2}$, integrating the last equality with respect to $\mathbb{P}_{2}$, gives that for every $x \in D, \mathbb{P}^{x}\left(Y_{S} \notin D, S<\infty\right)=\mathbb{P}^{x}\left(X_{T_{S}} \notin\right.$ $D, S<\infty)=\mathbb{P}^{x}(S<\infty)$. But this means that $S \geq \tau_{D}^{Y}$, $\mathbb{P}^{x}$-a.s. on $\{S<\infty\}$. Clearly, $S \geq \tau_{D}^{Y}$ on $\{S=\infty\}$.

Remark 3.3. Assume that $S_{n}<S$ for every $n \geq 1$. Note that in this case $Y_{S_{n}} \in D$. Since $S=\lim _{n \rightarrow \infty} S_{n}$, the quasi-left-continuity of $Y$ implies that on $\{S<\infty\}, Y_{S}=\lim _{n \rightarrow \infty} Y_{S_{n}} \in$ $\bar{D}$, the closure of D. From the proof of Proposition 3.2, we have that $Y_{S} \in D^{c}$ on $\{S<\infty\}$. Therefore, $Y_{S}=Y_{\tau_{D}^{Y}} \in \partial D$ on $\{S<\infty\}=\left\{\tau_{D}^{Y}<\infty\right\}$.

\section{Resurrection kernel}

Proposition 3.2 clearly shows that the process $Y^{D}$ can be obtained from $Z^{D}$ by resurrecting the latter at most countably many times. Our next goal is to compute the resurrection kernel. For $t \geq 0$ and $x \in E$, let $P_{t}(x, d y)$ denote the transition kernel of $X$. To be more precise, $P_{t}(x, d y)=\mathbb{P}_{1}^{x}\left(X_{t} \in d y\right)=\mathbb{P}^{x}\left(X_{t} \in d y\right)$. Similarly, for $x \in D$, let $P_{t}^{D}(x, d y)$ denotes the transition kernel of the killed process $X^{D}$. Throughout this paper we will assume the following (A1) $X$ admits a Lévy system of the form $\left(J^{X}, d t\right)$.

Here $J^{X}(x, d y)$ is a kernel on $(E, \mathcal{E})$. The assumption $(\mathbf{A 1})$ is not very restrictive. For example, all Lévy processes satisfy this assumption. Under the assumption (A1), one can easily check that the killed process $X^{D}$ has a Lévy system of the form $\left(J^{X^{D}}, d t\right)$, where $J^{X^{D}}(x, d y)$ is the restriction of $J^{X}$ on $(D, \mathcal{B}(D))$. That is, for $x \in D$ and a Borel subset $B \subset D, J^{X^{D}}(x, B)=J^{X}(x, B)$. By slightly abusing the notation, we will denote $J^{X^{D}}$ simply by $J^{X}$.

The subordinate process $Y$ admits a Lévy system of the form $\left(J^{Y}, d t\right)$, where

$$
J^{Y}(x, d y)=b J^{X}(x, d y)+\int_{(0, \infty)} P_{t}(x, d y) \Pi(d t), \quad x \in E,
$$

(see [8] for a proof, and also [3], p.74, for the case $b=0$ ).

Similarly, the subordinate killed process $Z^{D}$ admits a Lévy system of the form $\left(J^{Z^{D}}, d t\right)$, where

$$
J^{Z^{D}}(x, d y)=b J^{X}(x, d y)+\int_{(0, \infty)} P_{t}^{D}(x, d y) \Pi(d t), \quad x \in D
$$


It is also well known that the potential kernel $U^{Z^{D}}(x, d y)$ of $Z^{D}$ is given by

$$
U^{Z^{D}}(x, d y)=\int_{0}^{\infty} P_{t}^{D}(x, d y) U(d t)
$$

where $U(d s)$ is the potential measure of the subordinator $T$. Finally, we recall the following first-passage formulae for the subordinator (see, e.g., [1], p.76): For each fixed $x \geq 0$ and every $0 \leq s \leq x<t$

$$
\mathbb{P}_{2}\left(T_{\sigma_{x}-} \in d s, T_{\sigma_{x}} \in d t\right)=U(d s) \Pi(d t-s) .
$$

If $b=0$, then $\mathbb{P}_{2}\left(T_{\sigma_{x}}=x\right)=0$, while if $b>0$, then $U(d x)$ has a continuous density $u$ and

$$
\mathbb{P}_{2}\left(T_{\sigma_{x}}=x\right)=b u(x),
$$

(see, e.g., [1], pp.77-79). Moreover, $\mathbb{P}_{2}\left(T_{\sigma_{x}}=x\right)=\mathbb{P}_{2}\left(T_{\sigma_{x}-}=T_{\sigma_{x}}=x\right)$.

We are mainly interested in the case when the Lévy measure $\Pi$ of $T$ is infinite. So from now on, we assume

(A2) The Lévy measure $\Pi$ of $T$ is infinite.

In this case, the potential measure $U(d t)$ has no atoms (e.g. [6], Theorem 5.4).

Theorem 4.1. Suppose that (A1) and (A2) are valid. Let $B \subset \bar{D}$ and $C$ be Borel subsets of $E$. Then for every $x \in D$,

$$
\begin{aligned}
\mathbb{P}^{x}\left(Y_{\sigma_{\tau_{D}}} \in B, Y_{\sigma_{\tau_{D}}} \in C\right) & \\
= & \int_{B \cap D} U^{Z^{D}}(x, d y) \int_{C \cap D^{c}}\left(J^{Y}(y, d z)-b J^{X}(y, d z)\right) \\
& +\int_{B \cap D} U^{Z^{D}}(x, d y) \int_{C \cap D}\left(J^{Y}(y, d z)-J^{Z^{D}}(y, d z)\right) \\
& +b \mathbb{E}^{x}\left(u\left(\tau_{D}\right), X\left(\tau_{D}-\right) \in B, X\left(\tau_{D}\right) \in C \cap D^{c}\right) .
\end{aligned}
$$

Remark: In case when $b=0$, the last line vanishes.

Proof. Note that

$$
Y_{\sigma_{\tau_{D}}-}=\lim _{t \uparrow \sigma_{\tau_{D}}} Y_{t}=\lim _{t \uparrow \sigma_{\tau_{D}}} X\left(T_{t}\right)=X\left(T_{\sigma_{\tau_{D}}-}-\right)
$$

We first split the left-hand side in (4.6) depending on whether the subordinator jumps over, or hits, the level $\tau_{D}$ at the first passage over $\tau_{D}$ :

$$
\begin{aligned}
\mathbb{P}^{x}\left(Y_{\sigma_{\tau_{D}}} \in B, Y_{\sigma_{\tau_{D}}} \in C\right)= & \mathbb{P}^{x}\left(Y_{\sigma_{\tau_{D}}-} \in B, Y_{\sigma_{\tau_{D}}} \in C, T_{\sigma_{\tau_{D}}-} \leq \tau_{D}<T_{\sigma_{\tau_{D}}}\right) \\
& +\mathbb{P}^{x}\left(Y_{\sigma_{\tau_{D}}} \in B, Y_{\sigma_{\tau_{D}}} \in C, T_{\sigma_{\tau_{D}}}=\tau_{D}=T_{\sigma_{\tau_{D}}}\right) \\
=: & A_{1}+A_{2} .
\end{aligned}
$$


Next, $A_{1}$ can be written as

$$
\begin{aligned}
& A_{1}=\mathbb{P}^{x}\left(X\left(T_{\sigma_{\tau_{D}}-}-\right)\left(\omega_{1}, \omega_{2}\right) \in B, X\left(T_{\sigma_{\tau_{D}}}\right)\left(\omega_{1}, \omega_{2}\right) \in C,\right.
\end{aligned}
$$

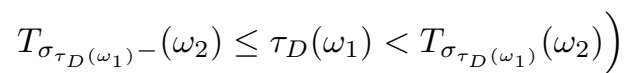

$$
\begin{aligned}
& =\left(\mathbb{P}_{1}^{x} \times \mathbb{P}_{2}\right)\left(X\left(T_{\sigma_{\tau_{D}\left(\omega_{1}\right)}-}\left(\omega_{2}\right)-\right)\left(\omega_{1}\right) \in B, X\left(T_{\sigma_{\tau_{D}\left(\omega_{1}\right)}}\left(\omega_{2}\right)\right)\left(\omega_{1}\right) \in C,\right. \\
& \left.\left.T_{\sigma_{\tau_{D}\left(\omega_{1}\right)}-}\left(\omega_{2}\right) \leq \tau_{D}\left(\omega_{1}\right)<T_{\sigma_{\tau_{D}\left(\omega_{1}\right)}}\left(\omega_{2}\right)\right)\right) \\
& =\mathbb{E}_{1}^{x}\left[\int_{0}^{\tau_{D}\left(\omega_{1}\right)} \int_{\tau_{D}\left(\omega_{1}\right)}^{\infty} \mathbb{P}_{2}\left(T_{\sigma_{\tau_{D}\left(\omega_{1}\right)}-} \in d s, T_{\sigma_{\tau_{D}\left(\omega_{1}\right)}} \in d t\right) 1_{\left(X_{s-}\left(\omega_{1}\right) \in B\right)} 1_{\left(X_{t}\left(\omega_{1}\right) \in C\right)}\right] \\
& =\mathbb{E}_{1}^{x} \int_{0}^{\tau_{D}} U(d s) \int_{\tau_{D}}^{\infty} \Pi(d t-s) 1_{\left(X_{s-} \in B\right)} 1_{\left(X_{t} \in C\right)} \\
& =\int_{0}^{\infty} U(d s) \mathbb{E}_{1}^{x}\left[1_{\left(s<\tau_{D}\right)} 1_{\left(X_{s} \in B\right)} \int_{\tau_{D}}^{\infty} \Pi(d t-s) 1_{\left(X_{t} \in C\right)}\right] \text {, }
\end{aligned}
$$

where the next to last line follows from (4.4), and the last line from the fact that $U$ has no atoms, and $X_{s-}=X_{s}, \mathbb{P}_{1}^{x}$-a.e. for every fixed $s \geq 0$. Next,

$$
\begin{aligned}
\mathbb{E}_{1}^{x} & {\left[1_{\left(s<\tau_{D}\right)} 1_{\left(X_{s} \in B\right)} \int_{\tau_{D}}^{\infty} \Pi(d t-s) 1_{\left(X_{t} \in C\right)}\right] } \\
= & \mathbb{E}_{1}^{x}\left[1_{\left(s<\tau_{D}\right)} 1_{\left(X_{s} \in B \cap D\right)} \mathbb{E}_{1}^{x}\left[\int_{\tau_{D}}^{\infty} \Pi(d t-s) 1_{\left(X_{t} \in C\right)} \mid \mathcal{F}_{s+}^{0}\right]\right] \\
= & \mathbb{E}_{1}^{x}\left[1_{\left(s<\tau_{D}\right)} 1_{\left(X_{s} \in B \cap D\right)} \mathbb{E}_{1}^{x}\left[\int_{s+\tau_{D} \circ \vartheta_{s}^{1}}^{\infty} \Pi(d t-s) 1_{\left(X_{t} \in C\right)} \mid \mathcal{F}_{s+}^{0}\right]\right] \\
= & \mathbb{E}_{1}^{x}\left[1_{\left(s<\tau_{D}\right)} 1_{\left(X_{s} \in B \cap D\right)} \mathbb{E}_{1}^{x}\left[\int_{\tau_{D} \circ \vartheta_{s}^{1}}^{\infty} \Pi(d w) 1_{\left(X_{w} \circ \vartheta_{s}^{1} \in C\right)} \mid \mathcal{F}_{s+}^{0}\right]\right] \\
= & \mathbb{E}_{1}^{x}\left[1_{\left(s<\tau_{D}\right)} 1_{\left(X_{s} \in B \cap D\right)} \mathbb{E}_{1}^{X_{s}} \int_{\tau_{D}}^{\infty} \Pi(d w) 1_{\left(X_{w} \in C\right)}\right] \\
= & \int_{B \cap D} P_{s}^{D}(x, d y) f(y),
\end{aligned}
$$

where

$$
\begin{aligned}
f(y) & =\mathbb{E}_{1}^{y}\left[\int_{\tau_{D}}^{\infty} \Pi(d w) 1_{\left(X_{w} \in C\right)}\right] \\
& =\int_{0}^{\infty} \Pi(d w) \mathbb{P}_{1}^{y}\left(X_{w} \in C, w \geq \tau_{D}\right)
\end{aligned}
$$

Since

$$
\begin{aligned}
\mathbb{P}_{1}^{y}\left(X_{w} \in C, w \geq \tau_{D}\right) & =\mathbb{P}_{1}^{y}\left(X_{w} \in C\right)-\mathbb{P}_{1}^{y}\left(X_{w} \in C, w<\tau_{D}\right) \\
& =\int_{C} P_{w}(y, d z)-\int_{C \cap D} P_{w}^{D}(y, d z)
\end{aligned}
$$


it follows from (4.1) and (4.2) that

$$
\begin{aligned}
f(y) & =\int_{C} \int_{0}^{\infty} \Pi(d w) P_{w}(y, d z)-\int_{C \cap D} \int_{0}^{\infty} \Pi(d w) P_{w}^{D}(y, d z) \\
& =\int_{C}\left(J^{Y}(y, d z)-b J^{X}(y, d z)\right)-\int_{C \cap D}\left(J^{Z^{D}}(y, d z)-b J^{X}(y, d z)\right) \\
& =\int_{C \cap D^{c}}\left(J^{Y}(y, d z)-b J^{X}(y, d z)\right)+\int_{C \cap D}\left(J^{Y}(y, d z)-J^{Z^{D}}(y, d z)\right) .
\end{aligned}
$$

Equalities (4.7), (4.8) and (4.9) yield that $A_{1}$ is equal to the first two lines on the right-hand side of (4.6).

In order to compute $A_{2}$ we proceed as follows:

$$
\begin{aligned}
A_{2} & =\mathbb{P}^{x}\left(Y_{\sigma_{\tau_{D}}} \in B, Y_{\sigma_{\tau_{D}}} \in C, T_{\sigma_{\tau_{D}}}=\tau_{D}=T_{\sigma_{\tau_{D}}}\right) \\
& =\mathbb{P}^{x}\left(X\left(\tau_{D}-\right) \in B, X\left(\tau_{D}\right) \in C, T_{\sigma_{\tau_{D}}}=\tau_{D}=T_{\sigma_{\tau_{D}}}\right) \\
& =\mathbb{E}_{1}^{x}\left[X\left(\tau_{D^{-}}\right)\left(\omega_{1}\right) \in B, X\left(\tau_{D}\right)\left(\omega_{1}\right) \in C, \mathbb{P}_{2}\left(T_{\sigma_{\tau_{D}\left(\omega_{1}\right)}}=\tau_{D}\left(\omega_{1}\right)\right)\right] \\
& =b \mathbb{E}^{x}\left[u\left(\tau_{D}\right), X\left(\tau_{D}-\right) \in B, X\left(\tau_{D}\right) \in C \cap D^{c}\right]
\end{aligned}
$$

where the last line follows from $(4.5)$ and the fact that $X\left(\tau_{D}\right) \notin D$.

For $y \in D$, let $q(y, d z):=J^{Y}(y, d z)-J^{Z^{D}}(y, d z)$. We call $q$ the resurrection kernel. Note that for Borel sets $B \subset D$ and $C \subset D$, the formula (4.6) can be written as

$$
\mathbb{P}^{x}\left(Y_{\sigma_{\tau_{D}}} \in B, Y_{\sigma_{\tau_{D}}} \in C\right)=\int_{B} U^{Z^{D}}(x, d y) q(y, C) .
$$

By use of (4.1) and (4.2) one can write the resurrection kernel as

$$
q(y, d z)=\int_{(0, \infty)}\left(P_{t}(y, d z)-P_{t}^{D}(y, d z)\right) \Pi(d t) .
$$

This is the form that the resurrection kernel appears in [5].

Corollary 4.2. Assume that (A1) and (A2) are valid.

(i) If the subordinator has no drift, i.e., $b=0$, then $\mathbb{P}^{x}\left(T_{\sigma_{\tau_{D}}}=\tau_{D}\right)=\mathbb{P}^{x}\left(T_{\sigma_{\tau_{D}}-}=\tau_{D}\right)=0$ and $\mathbb{P}^{x}\left(Y_{\sigma_{\tau_{D}}} \in D\right)=1$ for every $x \in D$.

(ii) If $b>0$, then for every Borel set $C \subset E$,

$$
\left.\mathbb{P}^{x}\left(Y_{\sigma_{\tau_{D}}} \in C, T_{\sigma_{\tau_{D}}}=\tau_{D}\right)=b \mathbb{E}^{x}\left[u\left(\tau_{D}\right), X\left(\tau_{D}\right) \in C \cap D^{c}\right)\right]
$$

\section{Proof.}

(i) That $\mathbb{P}^{x}\left(T_{\sigma_{\tau_{D}}}=\tau_{D}\right)=\mathbb{P}^{x}\left(T_{\sigma_{\tau_{D}}-}=\tau_{D}\right)=0$ is an immediate consequence of the first passage formulae stated before Theorem 4.1 and the fact that the potential measure $U$ has no atoms. In order to show that $\mathbb{P}^{x}\left(Y_{\sigma_{\tau_{D}}} \in D\right)=1$ we use (4.7):

$$
\begin{aligned}
\mathbb{P}^{x}\left(Y_{\sigma_{\tau_{D}}} \in D\right) & =\int_{0}^{\infty} U(d s) \mathbb{E}_{1}^{x}\left[1_{\left(s<\tau_{D}\right)} 1_{\left(X_{s} \in D\right)} \int_{\tau_{D}}^{\infty} \Pi(d t-s) 1_{\left(X_{t} \in E\right)}\right] \\
& =\int_{0}^{\infty} U(d s) \mathbb{E}_{1}^{x}\left[1_{\left(s<\tau_{D}\right)} \int_{\tau_{D}}^{\infty} \Pi(d t-s)\right] \\
& =\mathbb{E}_{1}^{x}\left[\int_{0}^{\tau_{D}} U(d s) \int_{\tau_{D}}^{\infty} \Pi(d t-s)\right]=1
\end{aligned}
$$


because the quantity in the last bracket is $\mathbb{P}_{1}^{x}$-a.s. equal to 1 .

(ii) This follows immediately from the expression for $A_{2}$ in the proof of Theorem 4.1 by taking $B=\bar{D}$.

Corollary 4.3. Suppose that (A1) and (A2) are valid. Assume that $J^{X}(x, \partial D)=0$ and $P_{t}(x, \partial D)=0$, for all $x \in D$ and all $t>0$. Then for every Borel subset $C \subset \partial D$,

$$
\mathbb{P}^{x}\left(Y_{\sigma_{\tau_{D}}} \in C\right)=b \mathbb{E}^{x}\left[u\left(\tau_{D}\right), X\left(\tau_{D}\right) \in C\right], \quad x \in D
$$

In particular, if $b=0, J^{X}(x, \partial D)=0$ and $P_{t}(x, \partial D)=0$, for all $x \in D$ and all $t>0$, then

$$
\mathbb{P}^{x}\left(Y_{\sigma_{\tau_{D}}} \in \partial D\right)=0
$$

Proof. It follows from (4.1) that $J^{Y}(x, \partial D)=0, x \in D$. Now (4.10) follows from Theorem 4.1 by taking $B=\bar{D}$ and $C=\partial D$, having (4.11) as an immediate consequence.

Corollary 4.4. Suppose that (A2) is valid. Assume that $X$ has continuous paths and $P_{t}(x, \partial D)=$ 0 for all $x \in D$ and all $t>0$. Then for every Borel set $C \subset \partial D$ it holds that

$$
\mathbb{P}^{x}\left(Y_{\sigma_{\tau_{D}}} \in C\right)=\mathbb{P}^{x}\left(Y_{\sigma_{\tau_{D}}} \in C\right)=b \mathbb{E}^{x}\left[u\left(\tau_{D}\right), X\left(\tau_{D}\right) \in C\right]
$$

In particular,

$$
\mathbb{P}^{x}\left(Y_{\sigma_{\tau_{D}}} \in \partial D\right)=\mathbb{P}^{x}\left(Y_{\sigma_{\tau_{D}}} \in \partial D\right)=b \mathbb{E}^{x}\left[u\left(\tau_{D}\right)\right]
$$

Proof. The second equality follows from Corollary 4.3. For the first, notice that by continuity of $X, Y_{\sigma_{\tau_{D}}}=X\left(T_{\sigma_{\tau_{D}}}\right)=X\left(T_{\sigma_{\tau_{D}}}\right)$. If $Y_{\sigma_{\tau_{D}}-} \in C \subset \partial D$, then $T_{\sigma_{\tau_{D}}}-=\tau_{D}=T_{\sigma_{\tau_{D}}}$ (see the proof of Corollary 4.2). Therefore, $Y_{\sigma_{\tau_{D}}}=Y_{\sigma_{\tau_{D}}}$.

\section{An application}

It is of some interest to find sufficient conditions for $Y_{\tau_{D}^{Y}} \notin \partial D$. In [11], Sztonyk gave a sufficient condition for a rotationally invariant Lévy process with infinite Lévy measure and no Gaussian component not to hit the boundary $\partial D$ upon exiting a Lipschitz domain $D$. The given condition is satisfied for a rotationally invariant processes. In this section we give two sufficient conditions in our setting.

For $t \geq 0$ and $x \in E$ define $N_{t}(x, f)=\mathbb{E}_{1}^{x}\left[f\left(X_{t}\right)\right]$, where $f$ is a nonnegative Borel function on $E$. Note that $T_{\sigma_{\tau_{D}}}=\tau_{D}+\left(T_{\sigma_{\tau_{D}}}-\tau_{D}\right)$, and for each fixed $\omega_{2}, T_{\sigma_{\tau_{D}}\left(\cdot, \omega_{2}\right)}\left(\omega_{2}\right)-\tau_{D}(\cdot)$ is $\mathcal{F}_{\tau_{D}}^{0}$-measurable. Therefore, by an extended version of the strong Markov property (see [2], pp.43-44), for each fixed $\omega_{2}$ (with $\omega_{2}$ suppressed in notation),

$$
\mathbb{E}_{1}^{x}\left[1_{D}\left(Y_{\sigma_{\tau_{D}}}\right) \mid \mathcal{F}_{\tau_{D}+}^{0}\right]=\mathbb{E}_{1}^{x}\left[1_{D}\left(X_{\tau_{D}+\left(T_{\sigma_{\tau_{D}}}-\tau_{D}\right)}\right) \mid \mathcal{F}_{\tau_{D}+}^{0}\right]=N_{T_{\sigma_{\tau_{D}}}-\tau_{D}}\left(X_{\tau_{D}}, 1_{D}\right), \quad \mathbb{P}_{1}^{x}-\text { a.s. }
$$


Proposition 5.1. Suppose that (A2) is valid. Assume that $X$ has continuous paths, $P_{t}(x, \partial D)=$ 0 for all $x \in D$ and all $t>0$, and that there exists a constant $c \in(0,1)$ such that

$$
\mathbb{P}_{1}^{x}\left(X_{t} \in D\right) \leq c \quad \text { for every } x \in \partial D \text { and every } t>0 .
$$

Assume further that the subordinator $T$ has no drift. Then $\mathbb{P}^{x}\left(Y_{\tau_{D}^{Y}} \in \partial D\right)=0$ for every $x \in D$.

Proof. By the assumptions, $X_{\tau_{D}} \in \partial D$ and $N_{t}\left(x, 1_{D}\right)=\mathbb{P}_{1}^{x}\left(X_{t} \in D\right) \leq c$, for all $x \in \partial D$ and all $t \geq 0$. By (5.1), this implies that for each fixed $\omega_{2}, \mathbb{P}_{1}^{x}\left(Y_{\sigma_{\tau_{D}}} \in D \mid \mathcal{F}_{\tau_{D}+}^{0}\right) \leq c, x \in D$, and therefore

$$
\mathbb{P}^{x}\left(Y_{\sigma_{\tau_{D}}} \in D\right) \leq c, \quad x \in D .
$$

Recall the notations $S_{1}=\sigma_{\tau_{D}}$, and for $n \geq 1, S_{n+1}=S_{n}+S_{1} \circ \theta_{S_{n}}$. By the strong Markov property of $Y$, it follows from (5.3) that

$$
\mathbb{P}^{x}\left(Y_{S_{n}} \in D\right) \leq c^{n}, \quad n \geq 1 .
$$

Let $N:=\inf \left\{n \geq 1: S_{n}=\tau_{D}^{Y}\right\}$ with the usual convention $\inf \emptyset=\infty$. It follows from the last displayed formula that $\mathbb{P}^{x}(N=\infty)=0$ for every $x \in D$. Hence, there are only finitely many $S_{n}$ which are less than $\tau_{D}^{Y}$. From Corollary 4.4, $\mathbb{P}^{x}\left(Y_{\sigma_{\tau_{D}}} \in \partial D\right)=\mathbb{P}^{x}\left(Y_{S_{1}} \in \partial D\right)=0$, and therefore by iteration $\mathbb{P}^{x}\left(Y_{S_{n}} \in \partial D\right)=0$ for all $n \in \mathbb{N}$ and all $x \in D$. Since $\tau_{Y}^{D}=S_{n}$ for some $n \in \mathbb{N}$, the claim of the proposition follows.

If $X$ is a Brownian motion in $\mathbb{R}^{d}$, then it was shown in [10] that (5.2) holds true provided $D$ is a bounded domain satisfying the exterior cone condition.

The next result should be compared to Lemma 1 from [12].

Proposition 5.2. Suppose that (A2) is valid. Assume that $X$ has continuous paths, $P_{t}(x, \partial D)=$ 0 for all $x \in D$ and all $t>0, b=0$, and $\sup _{x \in D} \mathbb{P}^{x}\left(Y_{\sigma_{\tau_{D}}} \in D\right)<1$. Then $\mathbb{P}^{x}\left(Y_{\tau_{D}^{Y}} \in \partial D\right)=0$ for every $x \in D$.

Proof. Again note that by Corollary 4.4, $\mathbb{P}^{x}\left(Y_{\sigma_{\tau_{D}}} \in \partial D\right)=0$. Therefore, if $Y_{\tau_{D}^{Y}} \in \partial D$, then $\sigma_{\tau_{D}}<\tau_{D}^{Y}$, and hence $Y_{\sigma_{\tau_{D}}} \in D$. Let $\gamma:=\sup _{x \in D} \mathbb{P}^{x}\left(Y_{\tau_{D}^{Y}} \in \partial D\right)$. By the strong Markov property of $Y$ at $\sigma_{\tau_{D}}$ and the assumptions we have

$$
\begin{aligned}
\mathbb{P}^{x}\left(Y_{\tau_{D}^{Y}} \in \partial D\right) & =\mathbb{P}^{x}\left(Y_{\tau_{D}^{Y}} \in \partial D, Y_{\sigma_{\tau_{D}}} \in D\right) \\
& =\mathbb{P}^{x}\left(\mathbb{P}^{Y_{\tau_{D}}}\left(Y_{\tau_{D}^{Y}} \in \partial D\right), Y_{\sigma_{\tau_{D}}} \in D\right) \\
& \leq \gamma \mathbb{P}^{x}\left(Y_{\sigma_{\tau_{D}}} \in D\right) .
\end{aligned}
$$

By taking the supremum over $x \in D$, it follows that $\gamma \leq \gamma \sup _{x \in D} \mathbb{P}^{x}\left(Y_{\sigma_{\tau_{D}}} \in D\right)$. Therefore, $\gamma=0$.

Acknowledgment: The second named author gratefully acknowledges the hospitality of Department of Mathematics at the University of Illinois at Urbana-Champaign where the research for this paper was done. 


\section{References}

[1] J. Bertoin, Levy Processes, Cambridge University Press, Cambridge, 1996. MR1406564

[2] R. M. Blumenthal, R. K. Getoor, Markov Processes and Potential Theory, Academic Press, New York, 1968. MR0264757

[3] N. Bouleau, Quelques résultats probabilistes sur la subordination au sens de Bochner, in Seminar on potential theory, Paris, No. 7, 54-81, Lecture Notes in Math., 1061, Springer, Berlin, 1984. MR0779295

[4] J. Glover, M. Rao, H. Šikić, R. Song, Г-potentials, in Classical and modern potential theory and applications (Chateau de Bonas, 1993), 217-232, Kluwer Acad. Publ., Dordrecht, 1994. MR1321619

[5] P. He, J. Ying, Subordinating the killed process versus killing the subordinate process, Proceedings Amer. Math. Soc. 135(2007), 523-529. MR2255299

[6] A. E. Kyprianou, Introductory Lectures on Fluctuations of Lévy Processes with Applications, Springer, Berlin, 2006. MR2250061

[7] P. A. Meyer, Renaisssance, recollements, mélanges, ralentissement de processus de Markov, Ann. Inst. Fourier 25(1975), 465-497. MR0415784

[8] A. Miyake, The subordination of Lévy system for Markov processes, Proc. Japan Acad. 45(1969), 601-604. MR0260019

[9] M. Sharpe, General Theory of Markov Processes, Academic Press, San Diego, 1988. MR0958914

[10] R. Song, Z. Vondraček, Potential theory of the killed Brownian motion in a domain, Probab. Theory Rel. Fields 125(2003), 578-592. MR1974415

[11] P. Sztonyk, On harmonic measure for Lévy processes, Probab. Math. Statist., 20 (2000), 383-390. MR1825650

[12] J.-M. Wu, Harmonic measures for symmetric stable processes, Studia Math. 149(2002), 281-293. MR1893056 